\title{
The Heritability of Corneal and Ocular Higher-Order Aberrations in Koreans: The Healthy Twin Study
}

\author{
Dong Hui Lim, ${ }^{1,2}$ Woori Kim, ${ }^{3}$ Gyule Han, ${ }^{1}$ Gi Hyun Bae, ${ }^{4}$ Myung Hun Kim, ${ }^{5}$ Myoung Joon Kim, ${ }^{6}$ \\ Yun-Mi Song, ${ }^{7}$ Eui-Sang Chung, ${ }^{1}$ Tae-Young Chung, ${ }^{1}$ and Joohon Sung ${ }^{3}$, for the Korean Healthy \\ Twin Study
}

\author{
${ }^{1}$ Department of Ophthalmology, Samsung Medical Center, Sungkyunkwan University School of Medicine, Seoul, South Korea \\ ${ }^{2}$ Department of Preventive Medicine, Catholic University School of Medicine, Seoul, South Korea \\ ${ }^{3}$ Department of Epidemiology, School of Public Health and Institute of Health and Environment, Seoul National University, Seoul, \\ Republic of Korea \\ ${ }^{4}$ Department of Ophthalmology, Sahmyook Medical Center, Seoul, South Korea \\ ${ }^{5}$ Saevit Eye Hospital, Goyang, South Korea \\ ${ }^{6}$ Department of Ophthalmology, Asan Medical Center, Ulsan University School of Medicine, Seoul, Republic of Korea \\ ${ }^{7}$ Department of Family Medicine, Samsung Medical Center, Sungkyunkwan University School of Medicine, Seoul, South Korea
}

Correspondence: Tae-Young Chung, Department of Ophthalmology, Samsung Medical Center, Sungkyunkwan University School of Medicine, 81 Irwon-Ro Gangnam-gu, Seoul, 135710 , South Korea; tychung@skku.edu. Joohon Sung, Department of Epidemiology, School of Public Health and Institute of Health and Environment, Seoul National University, 559 Gwanak-ro Gwanak-gu, 151-742, South Korea;

jsung@snu.ac.kr.

DHL and WK are joint first authors.

Submitted: September 27, 2014

Accepted: April 25, 2015

Citation: Lim DH, Kim W, Han G, et al.; for the Korean Healthy Twin Study. The heritability of corneal and ocular higher-order aberrations in Koreans: the Healthy Twin Study. Invest Ophthalmol Vis Sci. 2015;56:3919-3928. DOI:10.1167/iovs.14-15763
Purpose. To estimate the genetic influences on corneal and ocular higher-order aberrations (HoAs) in the Korean population.

Methods. This was a prospective, family-based twin cohort study. A total of 1272 adult twins and their family members, who were part of the Korean Healthy Twin Study from 2007-2011, were included. Corneal and ocular HoAs were measured. The genetic influences on HoAs were investigated by using variance-component methods after adjusting for age and sex. Narrow-sense heritability was calculated. Intraclass correlation coefficients (ICCs) were used to calculate degrees of resemblance among different types of family relationships.

Results. A total of 269 monozygotic (MZ) twin pairs (including $176 \mathrm{MZ}$ twin pairs and 93 orphan twins), 50 dizygotic (DZ) twin pairs (including $38 \mathrm{DZ}$ twin pairs and 12 orphan twins), and 739 adult first-degree relatives of twins in 358 families were included. For more than half of corneal HoAs, the narrow-sense heritability estimates were not significant. Horizontal coma was highly heritable among corneal HoAs. The ICCs of horizontal coma from MZ twin pairs, pooled first-degree pairs, and spouse pairs were $0.41,0.05$, and 0.00 , respectively. Among ocular HoAs, the estimated narrow-sense heritability of SA was 0.71 with the highest estimates. The ICCs of spherical aberration (SA) from MZ twins, pooled firstdegree pairs, and spouse pairs were $0.76,0.25$, and 0.06 , respectively.

Conclusions. Both corneal and ocular HoAs demonstrated smaller heritability. Corneal HoAs showed low heritability, suggesting individual environmental factors explain most of the variance of these HoAs in the Korean population. Ocular HoAs were moderately heritable.

Keywords: higher-order aberrations, heritability, twin study
$\mathrm{V}^{\mathrm{i}}$ isual quality may be influenced by higher-order aberrations. Therefore, such aberrations have attracted attention from ophthalmologists for decades. Correcting higher-order aberrations is considered one of the main approaches to improve optical quality; therefore, it is important to clearly identify common aberrations of the human eye. ${ }^{1}$ There are several ophthalmologic developments that correct higher-order aberrations, including wavefront-guided laser refractive surgery, aspherical intraocular lenses, and personalized contact lenses. These developments correct aberrations such as reduced contrast sensitivity, and relatively common night vision disturbances including glare and halos.

Previous studies have described ocular aberrations. ${ }^{2-7}$ However, most of these studies only explore the age-related changes and simple presentations of higher-order aberrations. Very few studies (with limited sample sizes) have investigated the genetic or environmental effects on higher-order aberra- tions. ${ }^{8,9}$ Yeh et al. ${ }^{8}$ have investigated the genetic effects on refractive errors and anterior corneal aberrations in 33 monozygotic (MZ) and 10 dizygotic (DZ) twin pairs. In the Genes in Myopia Twin Study, Dirani et al. ${ }^{9}$ evaluate the role of genetics in lower- and higher-order aberrations in 46 twin pairs. Further investigation is needed to understand the influences of both environmental and genetic factors on higher-order aberrations of human eyes.

In contrast, the heritability of refractive errors is well documented through a few large cohort and twin studies. ${ }^{10-15}$ There are both environmental and major genetic contributions to the development of myopia. ${ }^{16}$ We have previously investigated the heritability of myopia and ocular biometrics in Koreans in the Healthy Twin Study. ${ }^{17}$

Higher-order aberrations impact subjective visual satisfaction. It is preferable to examine ocular and corneal higher-order aberrations separately, because the crystalline lens and cornea 
are unique entities that can have their own optical aberrations. We investigated the genetic influences and heritability of ocular and corneal higher-order aberrations in the Korean population through the Healthy Twin Study.

\section{MeTHODS}

\section{Participants}

The participants were part of the Healthy Twin Study, a prospective cohort study that has recruited Korean adult twins and their family members through advertisements at public health agencies since 2005. Comprehensive ophthalmologic examinations and follow-up examinations were conducted in the Department of Ophthalmology at the Samsung Medical Center in Seoul, South Korea, since 2007. In total, 1688 individuals ( 665 men and 1023 women) underwent detailed eye examinations between 2007-2011. ${ }^{17}$ A more extensive explanation of the Healthy Twin Study has been published previously. ${ }^{17-19}$ There were 1344 individuals with corneal aberrations and 1262 individuals with ocular aberrations. Subjects were informed to discontinue contact lens wear and to bring their spectacles for ophthalmologic examinations. Contact lens wearers were excluded. The twin pair zygosities were determined with 16 short tandem repeat (STR) markers (15 autosomal markers and one sex-determining marker) for $67 \%$ of the pairs. The twin pair zygosities of $33 \%$ of pairs were unclear with the STR markers. Instead, a self-administered zygosity questionnaire was used, which has a positive predictive value of $97.2 \%$ for $\mathrm{MZ}$ and $95.0 \%$ for $\mathrm{DZ}$ twins. ${ }^{20}$ Written informed consent was obtained from all participants. The study was approved by the institutional review board of the Samsung Medical Center and all procedures adhered to the Declaration of Helsinki.

\section{Corneal Higher-Order Aberrations}

Exclusion criteria included a history of refractive surgery or of optical disorders. Of the original 1344 patients, 119 individuals who had undergone eye surgery (such as cataract surgery) or refractive surgery, including laser-assisted in situ keratomileusis (LASIK) or laser-assisted subepithelial keratomileusis (LASEK), were excluded from corneal high-order aberrations analysis. Additionally, 93 individuals who had a history of ocular disorders, such as strabismus, keratoconus, glaucoma, or cataracts, were also excluded. Corneal aberrations were adjusted for pupil diameter with VOL-CT software (Sarver \& Associates, Carbondale, IL, USA), and therefore pupil diameter was not considered in the exclusion criteria. The final sample size with corneal aberrations was 1132 participants including 475 men and 657 women with a mean age of $42.80 \pm 12.52$ (range, 17 79) years. Among these, there were $395 \mathrm{MZ}$ twin subjects (including $152 \mathrm{MZ}$ twin pairs and 91 orphan twins), $78 \mathrm{DZ}$ twin subjects (including $34 \mathrm{DZ}$ twin pairs and 10 orphan twins), and 659 singleton adult first-degree relatives of twins in 332 families. The distribution of first-degree relationships included 573 parent-offspring pairs (100 father-son pairs, 130 father-daughter pairs, 162 mother-son pairs, and 181 mother-daughter pairs), 234 nontwin sibling pairs (68 sister pairs, 43 brother pairs, and 123 sister-brother pairs), and 82 spouse pairs.

\section{Ocular Higher-Order Aberrations}

Participants with a history of refractive surgery or optical disorders were excluded. The excluded participants were 114 individuals who had undergone eye surgery (such as cataract surgery), refractive surgery including LASIK or LASEK, and 86 individuals with a history of ocular disorders such as strabismus, keratoconus, glaucoma, or cataracts. Participants ( $n=417$ ) with pupil diameters $<5.0 \mathrm{~mm}$ were also excluded. The final sample size for ocular aberrations was 645 participants including 275 men and 370 women with a mean age of $39.75 \pm 11.37$ (range, 17 78) years. There were 232 MZ twin subjects (including $72 \mathrm{MZ}$ twin pairs and 88 orphan twins), $51 \mathrm{DZ}$ twin subjects (including $15 \mathrm{DZ}$ twin pairs and 21 orphan twins), and 362 singleton adult first-degree relatives of the twins in 250 families. The first-degree relationships included 174 parent-offspring pairs ( 41 father-son pairs, 35 father-daughter pairs, 46 mother-son pairs, and 52 motherdaughter pairs), 106 nontwin sibling pairs (35 sister pairs, 19 brother pairs, and 52 sister-brother pairs), and 19 spouse pairs.

\section{Measurements}

Corneal aberrations were obtained from corneal topographic measurements. To obtain corneal aberration data, a converting program was used to measure corneal aberrations from corneal height measurements. In contrast, ocular higher-order aberrations were measured by standardized commercial aberrometry and showed the overall wavefront aberrations, including both corneal and lenticular components.

Participants underwent computed corneal topographic analysis (Orbscan IIz; Bausch \& Lomb, Buffalo, NY, USA). Corneal height data were computed by using conversion software (VOL-CT) for higher-order corneal aberrations. The VOL-CT program proposed by Thibos et al. ${ }^{21}$ allows to load wavefront analysis files from various corneal topography and is adopted as a standard for calculating and reporting optical aberrations of eyes. ${ }^{2,8,21-23}$ Ocular higher-order aberrations were measured by a wavefront aberrometer (WASCA; Carl Zeiss Meditec, Jena, Germany) under mesopic condition without cycloplegics, and the aberrometer reported the magnitude and sign of each higher-order aberrations by a string of Zernike coefficients. ${ }^{6}$ In general, each aberration map is expressed as the sum of basis functions, and the most common basis functions are the Zernike polynomials. Each Zernike coefficient was computed to root-mean-square (RMS) values by being squared and summed and then taking the square root. Zernike coefficients were scaled to a pupil diameter of $5.0 \mathrm{~mm}$ and corrected to a common wavelength of $550 \mathrm{~nm}$. Nondilated refraction measurements were made by an autorefractor (Topcon AT, Tokyo, Japan). The spherical equivalent was calculated by using the following standard formula: $\{$ spherical error + (cylindrical error/2)\}. Axial length was recorded by the corneal touch A-scan ultrasonography (Model 820; Allergan-Humphrey, San Leandro, CA, USA), after obtaining corneal topographic data and wavefront aberrometer measurements. Anterior chamber depth and corneal astigmatism were obtained with corneal topographic analysis (Orbscan IIz).

\section{Statistical Analysis}

The left eye was used for analysis because there was no significant difference in higher-order aberrations between right and left eyes $(P>0.05)$. The values of corneal and ocular higher-order aberrations were considered as a continuous scale. Higher-order aberrations were analyzed for both Zernike coefficients $\left(Z_{n}{ }^{m}\right)$ and RMS. The following ocular higher-order aberrations were included in the analysis: vertical coma $\left(Z_{3}{ }^{-1}\right)$, horizontal coma $\left(Z_{3}{ }^{1}\right)$, oblique trefoil $\left(Z_{3}{ }^{-3}\right)$, horizontal trefoil $\left(Z_{3}^{3}\right)$, spherical aberration $\left(Z_{4}^{0}\right)$ and RMS of coma $\left(Z_{3}{ }^{-1}\right.$ and $Z_{3}{ }^{1}$ combined), trefoil $\left(Z_{3}{ }^{-3}\right.$ and $Z_{3}{ }^{3}$ combined), and spherical aberration $\left(Z_{4}^{0}\right)$. The corneal higher-order aberrations were 
analyzed more specifically. The same methods used for ocular aberrations were used to calculate vertical coma $\left(Z_{3}^{-1}\right)$, horizontal coma $\left(Z_{3}{ }^{1}\right)$, oblique trefoil $\left(Z_{3}{ }^{-3}\right)$, horizontal trefoil $\left(Z_{3}{ }^{3}\right)$, spherical aberration $\left(Z_{4}^{0}\right)$ for corneal aberrations. Additionally, coma-like aberrations $\left(Z_{3}{ }^{-1}, Z_{3}{ }^{1}, Z_{5}{ }^{-1}, Z_{5}{ }^{1}\right.$ combined), spherical-like aberrations ( $Z_{4}{ }^{0}$ and $Z_{6}{ }^{0}$ combined), and higher-order aberrations from three to six orders were calculated. For the RMS values of corneal higher-order aberrations, coma $\left(Z_{3}{ }^{-1}\right.$ and $Z_{3}{ }^{1}$ combined), trefoil $\left(Z_{3}{ }^{-3}\right.$ and $Z_{3}{ }^{3}$ combined), and spherical aberration $\left(Z_{4}{ }^{0}\right)$ were calculated in the same way as were ocular aberrations. Additionally, RMS of coma-like aberrations $\left(Z_{3}{ }^{-1}, Z_{3}{ }^{1}, Z_{5}{ }^{-1}, Z_{5}{ }^{1}\right.$ combined), spherical-like aberrations $\left(Z_{4}^{0}\right.$ and $Z_{6}{ }^{0}$ combined), and third- to sixth-order aberrations were included. Higher-order aberrations were calculated by combining three to six orders. Total order aberrations were calculated by including lower-order and higher-order aberrations by combining zero to six orders. Since RMS values are positive values, log transformation was used to obtain a normal distribution. Student's $t$-test and analysis of variance were used to evaluate significant differences of higher-order aberrations between men and women and among age groups, respectively.

Intraclass correlation coefficients (ICCs) were used to investigate the degree of resemblance among different types of family relationships. Intraclass correlation coefficients were estimated for specific relationship types including MZ twins, DZ twins, sibling pairs, parent-offspring pairs, pooled pairs with DZ twins, sibling pairs, parent-offspring pairs, and spouse pairs. While MZ twins share the same genetic information, members of pooled pairs with all first-degree relatives share $50 \%$ of genetic information and spouse pairs do not share any genetic information. Thus, pooled first-degree relative pairs were separately analyzed. The ICCs were calculated as the proportion of covariance within a specific family relationship over the total variance, which is a sum of within-group and residual variance. The ICCs were calculated with a mixed model after testing normality and adjusting for age and sex in the nontwin pairs.

Variance-component methods were used to quantify the genetic contributions to higher-order aberrations. Extended families including twins and their family members were used to estimate heritability, ruling out the dominant genetic effects and considering all types of family relationships. ${ }^{24}$ Narrowsense heritability was calculated as the proportion of the variance explained by additive genetic effects over the total phenotypic variance (after adjusting for age, sex, age ${ }^{2}$, age-bysex interaction, and age $^{2}$-by-sex interaction). A high kurtosis was adjusted by assuming the multivariate $t$-distribution, and traits for which standard deviation is below 0.5 were transformed by multiplying appropriate values. ${ }^{25}$ The genetic models used include additive genetic effects, shared environment effects, and unique environment effects. The best-fitting model was selected on the basis of maximum likelihood.

Descriptive statistics and ICC calculations were carried out by using SAS (version 9.3; SAS, Inc., Cary, NC, USA). A variancecomponents model implemented in the Sequential Oligogenic Linkage Analysis Routines (SOLAR) software package (version 4.2.7; http://solar.txbiomedgenetics.org/; provided in the public domain by Texas Biomedical Research Institute) was used to calculate heritability.

\section{Results}

The basic characteristics of the study participants by age group are shown in Table 1. Biometrics such as the spherical equivalent, axial length, anterior chamber depth, and corneal astigmatism are also shown in Table 1. As age increased, the spherical equivalent was less myopic. Corneal astigmatism, axial length, and anterior chamber depth all decreased significantly with increased age $(P<0.0001)$. All biometrics were significantly different between males and females $(P<$ 0.0001). Corneal higher-order aberrations (except for spherical aberrations, spherical-like aberrations, and RMS values of both of them) were statistically different among age groups $(P<$ 0.05). While Zernike coefficients of corneal higher-order aberrations did not show a consistent change along with age groups, RMS-formed aberrations increased with older age groups consistently. Sex differences were found in corneal higher-order aberrations including horizontal trefoil, spherical aberration, spherical-like aberrations, RMS of spherical aberration, and RMS of spherical-like aberrations $(P<0.05)$. Ocular higher-order aberrations were statistically different among age groups $(P<0.05)$. Horizontal coma was not significantly different among age groups but increased slightly with increased age. Spherical aberration and RMS of spherical aberration in ocular higher-order aberrations were significantly different across the age groups $(P<0.0001)$, contrary to those in corneal higher-order aberrations. The sex difference of Zernike value and RMS of spherical aberration in both ocular and corneal higher-order aberrations were significant $(P<$ $0.05)$.

Intraclass correlation coefficients for corneal and ocular higher-order aberrations are presented in Table 2. Age and sex were adjusted where necessary. For the corneal higher-order aberrations, $152 \mathrm{MZ}$ twin pairs, $34 \mathrm{DZ}$ twin pairs, 234 sibling pairs, 573 parent-offspring pairs, and 82 spouse pairs were included in ICC analysis. There was a higher correlation of corneal higher-order aberrations among MZ twin pairs than there was in other family pairs. This finding suggests that there is a genetic influence on corneal higher-order aberrations. Interestingly, most of spherical aberration-related variables (including spherical aberration, spherical-like aberrations, fourth-order aberrations, sixth-order aberrations, RMS of spherical aberration, RMS of spherical-like aberrations, and RMS of fourth-order aberrations) showed relatively higher correlations among $\mathrm{MZ}$ twin pairs $(0.35,0.33,0.24,0.27,0.33$, 0.45 , and 0.27 , respectively) than they did among other family pairs (pooled first-degree relatives with DZ twins, siblings, and parent-offspring: $0.01,0.00,0.02,0.02,0.03,0.00$, and 0.07 , respectively; spouse: $0.02,0.19,0.01,0.01,0.04,0.11$, and 0.00 , respectively). For ocular higher-order aberrations, $72 \mathrm{MZ}$ twin pairs, $15 \mathrm{DZ}$ twin pairs, 106 sibling pairs, 174 parentoffspring pairs, and 19 spouse pairs were included. Ocular aberrations among MZ twin pairs showed higher ICCs than corneal aberrations among MZ twin pairs. Other than the RMS of trefoil, the ocular higher-order aberrations were highly correlated among MZ twins compared to other family pairs. The correlation of the RMS of trefoil in corneal higher-order aberrations (0.24) was much higher than it was in ocular higher-order aberrations (0.04) among MZ twin pairs. Spherical aberration had a correlation of 0.76 among $\mathrm{MZ}$ twin pairs, while those among other family pairs ranged from 0.37 to 0.55 . The narrow-sense heritability estimates for corneal and ocular higher-order aberrations are graphically shown in Figures 1 and 2 . Detailed descriptions of heritability estimates are included in Table 3. In general, the ocular higher-order aberrations had larger genetic effects than did the corneal higher-order aberrations. Most of the ocular higher-order aberrations were colored by yellow (heritability estimates $\left[b^{2}\right]$ ranging from $0.2-$ 0.4 ), while most of corneal higher-order aberrations were colored by gray ( $b^{2}$ under 0.2 ). In other words, corneal higherorder aberrations are more influenced by environmental effects than are ocular higher-order aberrations.

With regard to the corneal higher-order aberrations, there were statistically significant heritability estimates found in 
TABLE 1. Basic Characteristics of Participants in the Healthy Twin Study

\begin{tabular}{|c|c|c|c|c|c|c|c|c|}
\hline \multirow[b]{2}{*}{ Trait } & \multicolumn{5}{|c|}{ Age Group, y } & \multirow[b]{2}{*}{$P$ Value* } & \multicolumn{2}{|c|}{ Total } \\
\hline & $\sim 29$ & $30 \sim 39$ & $40 \sim 49$ & $50 \sim 59$ & $60 \sim$ & & Male & Female \\
\hline \multicolumn{9}{|l|}{ Biometrics } \\
\hline Spherical equivalent, D & $-2.77(2.18)$ & $-1.84(2.19)$ & $-1.07(1.83)$ & $-0.45(2.36)$ & $0.65(1.61)$ & $<0.0001$ & $-1.03(2.01)$ & $-1.39(2.46)$ \\
\hline Axial length, mm & $24.64(1.28)$ & $24.29(1.29)$ & $23.58(1.23)$ & $23.67(1.29)$ & $23.44(0.79)$ & $<0.0001$ & $24.29(1.16)$ & $23.79(1.34)$ \\
\hline Anterior chamber depth, $\mathrm{mm}$ & $3.18(0.29)$ & $2.95(0.35)$ & $2.78(0.41)$ & $2.60(0.37)$ & $2.57(0.53)$ & $<0.0001$ & $2.92(0.45)$ & $2.77(0.40)$ \\
\hline Corneal astigmatism, D & $1.27(0.85)$ & $0.97(0.67)$ & $0.83(0.78)$ & $0.78(0.55)$ & $0.80(0.52)$ & $<0.0001$ & $0.86(0.73)$ & $0.97(0.69)$ \\
\hline \multicolumn{9}{|l|}{ Corneal aberration, $\mu \mathrm{m}$} \\
\hline$N$ & 147 & 363 & 299 & 185 & 138 & & 475 & 657 \\
\hline \multicolumn{9}{|l|}{ Zernike coefficients } \\
\hline Vertical coma & $-0.03(0.14)$ & $-0.01(0.16)$ & $-0.04(0.17)$ & $-0.01(0.18)$ & $0.04(0.19)$ & $<0.0001$ & $-0.02(0.17)$ & $-0.01(0.18)$ \\
\hline Horizontal coma & $0.01(0.13)$ & $0.02(0.12)$ & $0.00(0.12)$ & $-0.03(0.13)$ & $-0.01(0.16)$ & 0.001 & $0.00(0.12)$ & $0.00(0.13)$ \\
\hline Oblique trefoil & $0.01(0.10)$ & $0.02(0.10)$ & $0.01(0.11)$ & $0.02(0.13)$ & $0.04(0.13)$ & 0.063 & $0.01(0.11)$ & $0.02(0.11)$ \\
\hline Horizontal trefoil & $-0.01(0.09)$ & $-0.02(0.08)$ & $-0.04(0.09)$ & $-0.04(0.11)$ & $-0.05(0.11)$ & $<0.0001$ & $-0.04(0.09)$ & $-0.03(0.09)$ \\
\hline Spherical aberration & $0.06(0.03)$ & $0.06(0.03)$ & $0.06(0.03)$ & $0.05(0.04)$ & $0.05(0.04)$ & 0.600 & $0.05(0.04)$ & $0.06(0.04)$ \\
\hline Coma-like aberrations & $-0.02(0.11)$ & $-0.02(0.11)$ & $-0.05(0.11)$ & $-0.05(0.13)$ & $-0.01(0.17)$ & 0.001 & $-0.04(0.12)$ & $-0.03(0.13)$ \\
\hline Spherical-like aberrations & $0.05(0.04)$ & $0.05(0.04)$ & $0.06(0.04)$ & $0.06(0.04)$ & $0.06(0.05)$ & 0.159 & $0.05(0.04)$ & $0.06(0.04)$ \\
\hline Third-order aberrations & $-0.01(0.25)$ & $0.00(0.27)$ & $-0.08(0.30)$ & $-0.05(0.34)$ & $0.02(0.37)$ & 0.001 & $-0.05(0.29)$ & $-0.02(0.31)$ \\
\hline Fourth-order aberrations & $0.04(0.09)$ & $0.02(0.11)$ & $0.03(0.11)$ & $0.05(0.13)$ & $0.04(0.13)$ & 0.047 & $0.03(0.12)$ & $0.03(0.11)$ \\
\hline Fifth-order aberrations & $-0.02(0.14)$ & $-0.04(0.16)$ & $-0.01(0.17)$ & $-0.04(0.19)$ & $-0.07(0.20)$ & 0.001 & $-0.02(0.17)$ & $-0.04(0.17)$ \\
\hline Sixth-order aberrations & $0.01(0.05)$ & $0.02(0.06)$ & $0.03(0.06)$ & $0.01(0.07)$ & $0.00(0.09)$ & 0.000 & $0.02(0.06)$ & $0.02(0.06)$ \\
\hline HoA & $0.02(0.17)$ & $0.00(0.18)$ & $-0.04(0.22)$ & $-0.04(0.24)$ & $-0.01(0.28)$ & 0.026 & $-0.03(0.21)$ & $-0.01(0.22)$ \\
\hline \multicolumn{9}{|l|}{ Root-mean-square } \\
\hline Coma & $0.16(0.11)$ & $0.17(0.11)$ & $0.18(0.11)$ & $0.20(0.11)$ & $0.22(0.12)$ & $<0.0001$ & $0.18(0.11)$ & $0.19(0.11)$ \\
\hline Trefoil & $0.11(0.08)$ & $0.12(0.07)$ & $0.12(0.08)$ & $0.15(0.09)$ & $0.15(0.09)$ & $<0.0001$ & $0.13(0.08)$ & $0.13(0.08)$ \\
\hline Spherica & $0.06(0.03)$ & $0.06(0.03)$ & $0.06(0.03)$ & $0.06(0.04)$ & $0.06(0.04)$ & 0.512 & $0.057(0.03)$ & $0.061(0.03)$ \\
\hline Coma-like aberrations & $0.19(0.11)$ & $0.20(0.12)$ & $0.21(0.12)$ & $0.23(0.12)$ & $0.26(0.14)$ & $<0.0001$ & $0.20(0.12)$ & $0.21(0.13)$ \\
\hline Spherical-like aberrations & $0.06(0.03)$ & $0.06(0.03)$ & $0.07(0.03)$ & $0.07(0.04)$ & $0.06(0.04)$ & 0.508 & $0.06(0.03)$ & $0.07(0.03)$ \\
\hline Third-order aberrations & $0.21(0.12)$ & $0.22(0.11)$ & $0.23(0.12)$ & $0.26(0.12)$ & $0.28(0.13)$ & $<0.0001$ & $0.23(0.11)$ & $0.24(0.13)$ \\
\hline Fourth-order aberrations & $0.10(0.04)$ & $0.11(0.04)$ & $0.11(0.04)$ & $0.12(0.05)$ & $0.13(0.05)$ & $<0.0001$ & $0.11(0.05)$ & $0.11(0.04)$ \\
\hline Fifth-or & $0.12(0.07)$ & $0.13(0.08)$ & $0.13(0.08)$ & $0.15(0.09)$ & $0.17(0.09)$ & $<0.0001$ & $0.14(0.08)$ & $0.14(0.08)$ \\
\hline Sixth-or & $0.06(0.03)$ & $0.06(0.03)$ & $0.07(0.03)$ & $0.07(0.03)$ & $0.08(0.04)$ & $<0.0001$ & $0.07(0.03)$ & $0.07(0.03)$ \\
\hline HoA & $0.27(0.13)$ & $0.29(0.13)$ & $0.30(0.14)$ & $0.34(0.15)$ & $0.37(0.15)$ & $<0.0001$ & $0.31(0.13)$ & $0.31(0.15)$ \\
\hline \multicolumn{9}{|l|}{ Ocular aberration, $\mu \mathrm{m}$} \\
\hline$N$ & 108 & 249 & 151 & 97 & 40 & & 275 & 370 \\
\hline \multicolumn{9}{|l|}{ Zernike coefficients } \\
\hline Vertic & $0.04(0.19)$ & $-0.01(0.20)$ & $-0.05(0.29)$ & $-0.12(0.24)$ & $-0.20(0.30)$ & $<0.0001$ & $-0.03(0.27)$ & $-0.05(0.23)$ \\
\hline Horizont & $-0.15(0.29)$ & $-0.12(0.32)$ & $-0.08(0.34)$ & $-0.10(0.38)$ & $0.02(0.41)$ & 0.086 & $-0.10(0.33)$ & $-0.10(0.34)$ \\
\hline Oblique trefoil & $-0.07(0.22)$ & $-0.04(0.21)$ & $0.04(0.30)$ & $0.02(0.26)$ & $0.07(0.32)$ & 0.0002 & $0.01(0.29)$ & $-0.02(0.22)$ \\
\hline Horizontal trefoil & $-0.07(0.27)$ & $-0.11(0.27)$ & $-0.15(0.26)$ & $-0.18(0.36)$ & $-0.09(0.39)$ & 0.041 & $-0.12(0.29)$ & $-0.13(0.30)$ \\
\hline Spherical aberration & $-0.07(0.16)$ & $-0.10(0.15)$ & $-0.15(0.19)$ & $-0.18(0.18)$ & $-0.17(0.18)$ & $<0.0001$ & $-0.10(0.15)$ & $-0.14(0.18)$ \\
\hline \multicolumn{9}{|l|}{ Root-mean-square } \\
\hline Coma & $0.32(0.19)$ & $0.33(0.21)$ & $0.38(0.25)$ & $0.41(0.25)$ & $0.48(0.24)$ & $<0.0001$ & $0.36(0.24)$ & $0.36(0.22)$ \\
\hline Trefoil & $0.30(0.20)$ & $0.31(0.18)$ & $0.34(0.26)$ & $0.40(0.26)$ & $0.45(0.26)$ & $<0.0001$ & $0.34(0.25)$ & $0.33(0.21)$ \\
\hline Spherical aberration & $0.14(0.11)$ & $0.14(0.11)$ & $0.19(0.15)$ & $0.21(0.15)$ & $0.20(0.14)$ & $<0.0001$ & $0.14(0.11)$ & $0.19(0.14)$ \\
\hline HoA & $0.21(0.08)$ & $0.21(0.08)$ & $0.24(0.11)$ & $0.27(0.09)$ & $0.30(0.08)$ & $<0.0001$ & $0.23(0.10)$ & $0.24(0.09)$ \\
\hline Total aberration & $2.96(1.99)$ & $2.00(1.65)$ & $1.61(1.54)$ & $1.40(1.38)$ & $1.11(0.57)$ & $<0.0001$ & $1.85(1.50)$ & $1.98(1.81)$ \\
\hline
\end{tabular}

Mean (SD).

${ }^{*} P$ value of age group difference.

vertical coma, horizontal coma, horizontal trefoil, spherical aberration, spherical-like aberrations, fourth-order aberrations, sixth-order aberrations, RMS of spherical aberration, RMS of spherical-like aberrations, and RMS of fourth-order aberrations $(P<0.05)$. These findings were identified with the AE (additive genetic effects and unique environmental effects) model as the best-fitting model. Although the ACE (additive genetic effects, common environmental effects and unique environmental effects) model was revealed in oblique trefoil, coma-like aberrations, third-order aberrations, fifth-order aberrations, all higher-order aberrations, and RMS of trefoil as the best-fitting model, both components of additive genetic effects and shared environmental effects did not show statistical significance of $P$ $<0.05$. Particularly, spherical aberration-related variables showed significant heritability estimates: spherical aberrations (0.18), spherical-like aberrations (0.12), fourth-order aberrations (0.16), sixth-order aberrations (0.15), RMS of spherical aberrations (0.22), RMS of spherical-like aberrations (0.17), and RMS of fourth-order aberrations (0.19). Horizontal coma had the highest heritability estimates of $b^{2}=0.31$ among the corneal higher-order aberrations. 
TABLE 2. Intraclass Correlation Coefficients for Corneal and Ocular Higher-Order Aberrations

\begin{tabular}{|c|c|c|c|c|c|c|c|}
\hline \multirow[b]{2}{*}{ Trait } & \multirow[b]{2}{*}{ MZ } & \multicolumn{5}{|c|}{ First-Degree Relatives } & \multirow[b]{2}{*}{ Spouse } \\
\hline & & $\mathbf{D Z}$ & $\mathbf{S B}^{*}$ & $\mathbf{D Z}+\mathbf{S B}^{*}$ & PO* & $\mathbf{D Z}+\mathbf{S B}+\mathbf{P O}^{*}$ & \\
\hline \multicolumn{8}{|l|}{ Corneal aberration, $\mu \mathrm{m}$} \\
\hline Pair & 152 & 34 & 234 & 339 & 573 & 912 & 82 \\
\hline \multicolumn{8}{|l|}{ Zernike coefficients } \\
\hline Vertical coma & 0.07 & 0.05 & 0.15 & 0.12 & 0.09 & 0.11 & 0.00 \\
\hline Horizontal coma & 0.41 & 0.23 & 0.00 & 0.04 & 0.05 & 0.05 & 0.00 \\
\hline Oblique trefoil & 0.05 & 0.09 & 0.00 & 0.03 & 0.05 & 0.05 & 0.07 \\
\hline Horizontal trefoil & 0.20 & 0.07 & 0.15 & 0.10 & 0.09 & 0.11 & 0.14 \\
\hline Spherical aberration & 0.35 & 0.00 & 0.06 & 0.04 & 0.00 & 0.01 & 0.02 \\
\hline Coma-like aberrations & 0.15 & 0.09 & 0.13 & 0.12 & 0.00 & 0.03 & 0.02 \\
\hline Spherical-like aberrations & 0.33 & 0.00 & 0.02 & 0.00 & 0.00 & 0.00 & 0.19 \\
\hline Third-order aberrations & 0.00 & 0.05 & 0.06 & 0.06 & 0.00 & 0.03 & 0.05 \\
\hline Fourth-order aberrations & 0.24 & 0.00 & 0.12 & 0.08 & 0.00 & 0.02 & 0.01 \\
\hline Fifth-order aberrations & 0.06 & 0.01 & 0.01 & 0.03 & 0.08 & 0.07 & 0.01 \\
\hline Sixth-order aberrations & 0.27 & 0.19 & 0.05 & 0.05 & 0.01 & 0.02 & 0.01 \\
\hline HoA & 0.12 & 0.03 & 0.04 & 0.04 & 0.01 & 0.02 & 0.16 \\
\hline \multicolumn{8}{|l|}{ Root-mean-square } \\
\hline Coma & 0.11 & 0.00 & 0.09 & 0.07 & 0.06 & 0.06 & 0.00 \\
\hline Trefoil & 0.24 & 0.00 & 0.12 & 0.07 & 0.01 & 0.03 & 0.00 \\
\hline Spherical aberration & 0.33 & 0.00 & 0.08 & 0.09 & 0.00 & 0.03 & 0.04 \\
\hline Coma-like aberrations & 0.09 & 0.00 & 0.11 & 0.07 & 0.08 & 0.08 & 0.00 \\
\hline Spherical-like aberrations & 0.45 & 0.00 & 0.00 & 0.00 & 0.00 & 0.00 & 0.11 \\
\hline Third-order aberrations & 0.14 & 0.00 & 0.12 & 0.07 & 0.02 & 0.04 & 0.00 \\
\hline Fourth-order aberrations & 0.27 & 0.00 & 0.00 & 0.03 & 0.08 & 0.07 & 0.00 \\
\hline Fifth-order aberrations & 0.15 & 0.00 & 0.02 & 0.00 & 0.04 & 0.03 & 0.00 \\
\hline Sixth-order aberrations & 0.02 & 0.00 & 0.06 & 0.02 & 0.08 & 0.08 & 0.00 \\
\hline HoA & 0.14 & 0.00 & 0.10 & 0.05 & 0.03 & 0.05 & 0.00 \\
\hline \multicolumn{8}{|l|}{ Ocular aberration, $\mu \mathrm{m}$} \\
\hline Pair & 72 & 15 & 106 & 157 & 174 & 331 & 19 \\
\hline \multicolumn{8}{|l|}{ Zernike coefficients } \\
\hline Vertical coma & 0.55 & 0.28 & 0.10 & 0.15 & 0.01 & 0.09 & 0.00 \\
\hline Horizontal coma & 0.43 & 0.25 & 0.15 & 0.14 & 0.00 & 0.06 & 0.00 \\
\hline Oblique trefoil & 0.46 & 0.20 & 0.21 & 0.17 & 0.15 & 0.17 & 0.00 \\
\hline Horizontal trefoil & 0.37 & 0.00 & 0.13 & 0.16 & 0.19 & 0.17 & 0.30 \\
\hline Spherical aberration & 0.76 & 0.57 & 0.24 & 0.27 & 0.25 & 0.25 & 0.06 \\
\hline \multicolumn{8}{|l|}{ Root-mean-square } \\
\hline Coma & 0.32 & 0.00 & 0.07 & 0.00 & 0.03 & 0.02 & 0.00 \\
\hline Trefoil & 0.04 & 0.04 & 0.11 & 0.13 & 0.00 & 0.05 & 0.00 \\
\hline Spherical aberration & 0.54 & 0.13 & 0.00 & 0.00 & 0.00 & 0.00 & 0.00 \\
\hline HoA & 0.37 & 0.00 & 0.16 & 0.11 & 0.10 & 0.11 & 0.00 \\
\hline Total aberration & 0.74 & 0.42 & 0.31 & 0.41 & 0.00 & 0.19 & 0.07 \\
\hline
\end{tabular}

PO, parent-offspring; SB, siblings.

$*$ Adjusted for age and sex.

All ocular higher-order aberrations (except for RMS of coma and RMS of trefoil) had a heritability that was statistically significantly greater than zero $(P<0.001)$. Spherical aberration produced the highest heritability estimate of 0.71 compared to those of the other types of ocular higher-order aberrations, which ranged from 0.31 to 0.39 .

\section{Discussion}

We comprehensively investigated the heritability of both corneal and ocular higher-order aberrations in the Korean population. The overall heritability of higher-order aberrations was much lower than that of lower-order aberrations. We had previously found that all traits, including spherical equivalent, axial length, anterior chamber depth, and corneal astigmatism, are highly heritable. ${ }^{17}$ Therefore, we can infer that lower-order aberrations are highly influenced by genetic factors. In this current study, we found that higher-order aberrations are less strongly heritable than are lower-order aberrations. The difference in heritability between lower- and higher-order aberrations means more variation is explained by environmental factors. We found that ocular higher-order aberrations were more heritable than were corneal higher-order aberrations. Spherical aberration-related variables showed moderate heritability estimates in both corneal and ocular higher-order aberrations, ranging from 0.12 to 0.71 . There were also statistically significant differences between males and females in spherical aberration-related variables. Both corneal and ocular higher-order aberrations increased with age. This trend is true for all aberrations except for corneal spherical aberration-related variables and is consistent with previous findings. ${ }^{2-4,7}$ Our study not only analyzed the higher-order 


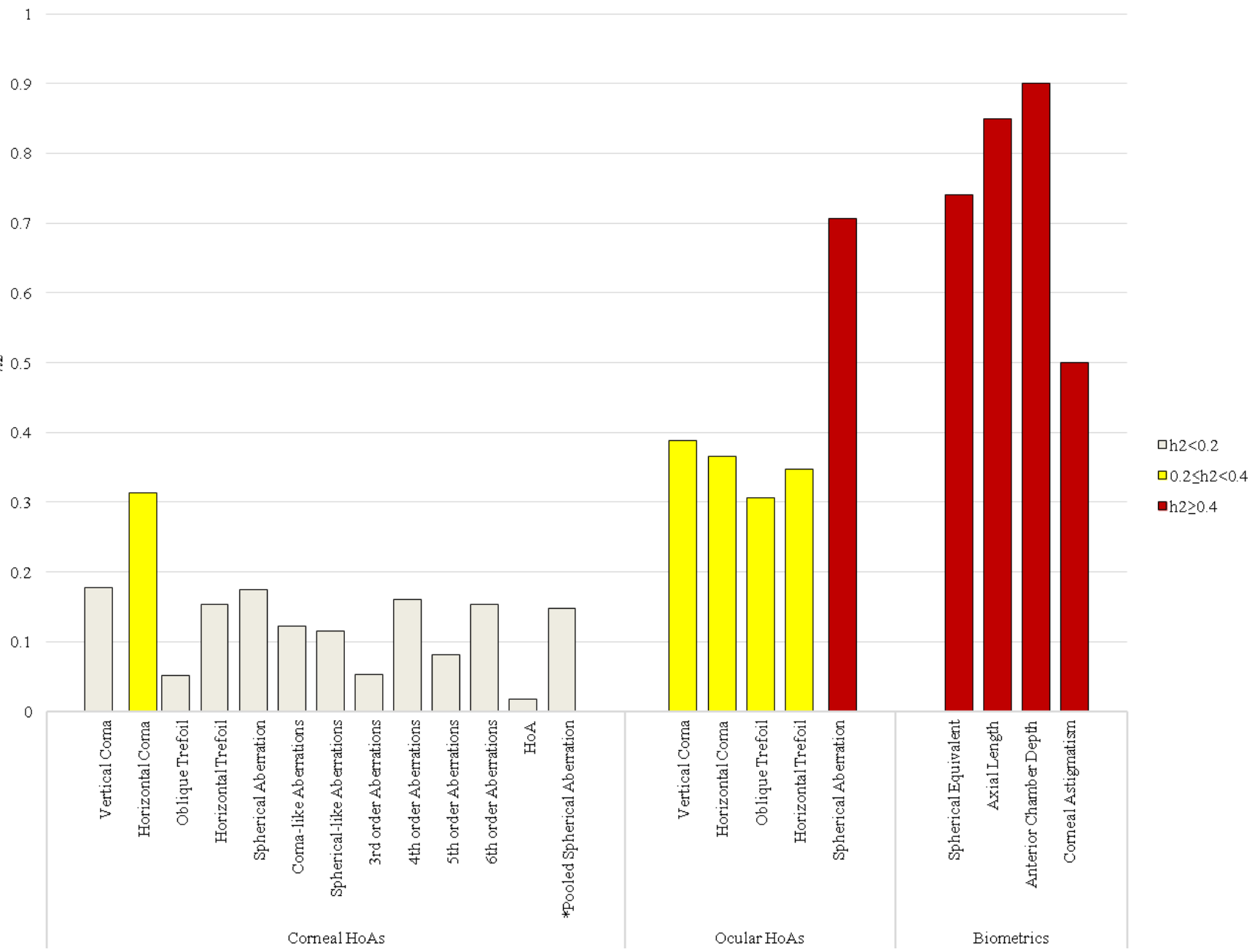

Figure 1. Heritability estimates for corneal and ocular higher-order aberrations of Zernike coefficients. *Pooled spherical aberration: mean of heritability of spherical aberration and spherical-like aberrations. $\boldsymbol{b}^{2}$, narrow-sense heritability estimates.

aberrations of the cornea and the whole eye in more detail, but also was larger in sample size than were other studies.

Few past studies have investigated the genetic influence on higher-order aberrations. No previous studies have evaluated both corneal and ocular higher-order aberrations. In the Twin Eye Study, Yeh et al. ${ }^{8}$ have investigated the genetic effects on refractive errors and anterior corneal aberrations in $33 \mathrm{MZ}$ twin pairs with mean age of $36.47 \pm 1.64$ years and $10 \mathrm{DZ}$ twin pairs with mean age of $37.54 \pm 2.60$ years. They find that the heritability estimate of corneal spherical aberration is 0.56 , which is higher than that of other corneal aberrations. We found a corresponding heritability estimate of 0.18 . This discrepancy may be attributable to the smaller sample size and the different estimation method used in the previous study. The classic twin models could cause the heritability estimate to be inflated. In the Genes in Myopia Twin Study, Dirani et al. ${ }^{9}$ evaluated the genetic influence on lower- and higher-order aberrations with a total of $32 \mathrm{MZ}$ and $14 \mathrm{DZ}$ twin pairs with a mean age of 65.3 years. ${ }^{9}$ All ocular higher-order aberrations are found to have higher intrapair correlations in MZ twins than in DZ twins, implying potential genetic influences; intrapair correlations of $\mathrm{MZ}$ for coma, trefoil, and quatrefoil exceed twice that of DZ, suggesting the role of nonadditive genetic effects. The study estimates the crude heritability estimates of ocular spherical aberration to be 0.08 , which is much smaller than our finding of 0.71 . However, the previous study does not reach statistical significance between intrapair correlations in MZ and DZ twin pairs. This discrepancy may again be explained by a smaller sample size in the previous study.

As explained above, the discrepancies in heritability results found between prior studies and the current study may be due to different sample sizes and analyses used. Moreover, ethnic differences have been reported in the distributions of higherorder aberrations in human eyes. ${ }^{26-30}$ We used the same method as we had priorly to calculate the heritability of myopia to quantify the genetic contributions to higher-order aberrations. ${ }^{17}$ Dominant genetic effects were eliminated by using the extended families, including twins and their family members, in our analyses. ${ }^{24}$ After adjusting for age and sex, narrow-sense heritability was calculated as the proportion of variance explained by additive genetic effects over the total phenotypic variance. The best-fitting genetic model was selected on the basis of maximum likelihood. In the previous studies, the genetic influences have been calculated with the equation $(r \mathrm{MZ}-r \mathrm{DZ}) \times 2$, where $r$ is the correlation coefficient. The use of this equation, along with smaller sample sizes, may overestimate heritability compared to narrow-sense heritability.

We found that the genetic influence on higher-order aberrations is considerably different between corneal and ocular aberrations. While corneal aberrations are relatively dependent on environmental effects, ocular aberrations are 


$$
1
$$$$
0.9-8 \longrightarrow
$$$$
0.7-6
$$$$
\text { ₹ู } 0.5
$$$$
0.4
$$

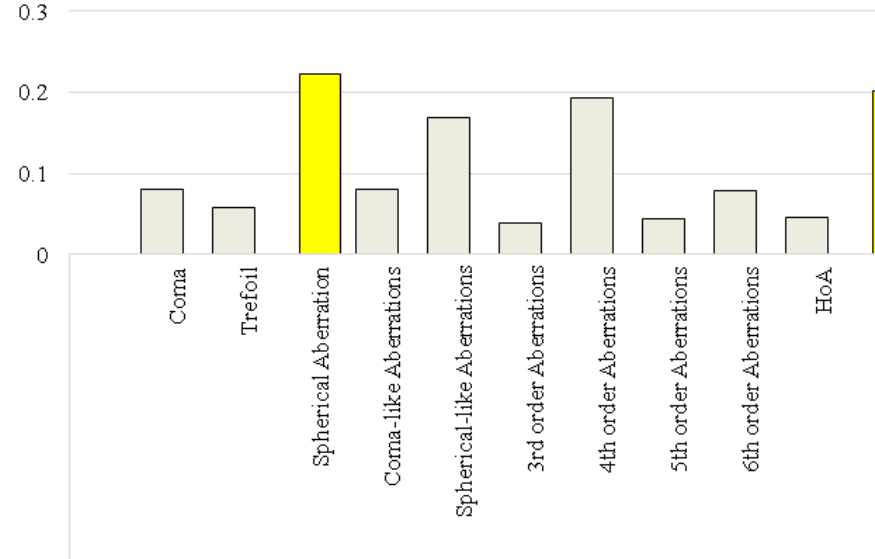

Corneal HoAs

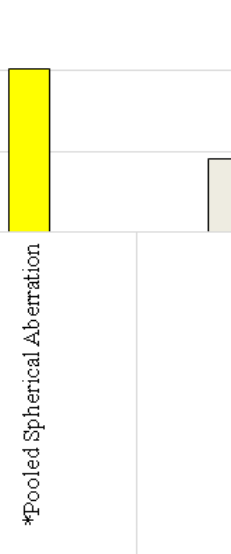

$\square \mathrm{h} 2<0.2$

$\square 0.2 \leq \mathrm{h} 2<0.4$

$\square \mathrm{h} \geq 0.4$

Figure 2. Heritability estimates for corneal and ocular higher-order aberrations of root-mean-square. *Pooled spherical aberration: mean of heritability of spherical aberration and spherical-like aberrations.

moderately heritable with heritability estimates ranging from 0.31 to 0.71 . More than half of the heritability estimates of corneal higher-order aberrations were not significant, while most ocular heritability estimates were strongly significant $(P$ $<0.001)$. Even those corneal higher-order aberrations that had significant heritability estimates were lower than those of ocular higher-order aberrations. The estimated narrow-sense heritability of horizontal coma was $0.31 \pm 0.06$ with the highest estimates among corneal aberrations. The ICCs of horizontal coma from MZ twin pairs, pooled first-degree pairs, and spouse pairs were $0.41,0.05$, and 0.00 , respectively. Among ocular aberrations, the estimated narrow-sense heritability of spherical aberration was highest at $0.71 \pm 0.06$. The ICCs of spherical aberration from MZ twin pairs, pooled firstdegree pairs, and spouse pairs were 0.76, 0.25, and 0.06, respectively.

There are several reasons that may explain the much lower heritability in corneal higher-order aberrations than in ocular higher-order aberrations. The anterior corneal surface may be the part of the eye that is most susceptible to physical change by environmental factors. Little is known about the risk factors that influence the shape of the cornea, except the tear film. The "E," unique environmental factor, is more likely to be random or error or chance, which is not explained by any covariates of this study. Random effect, including measurement errors, can possibly affect the lower heritability of corneal higher-order aberrations. Although Orbscan IIz is accepted as a gold standard to map the corneal anterior surface, environmental effect, including tear film instability, surface irregularity, light scattering, and microsaccades, may give rise to measure- ment errors. ${ }^{8,31-33}$ Changes in the tear film may especially influence the corneal higher-order aberrations. Several prior studies have reported that disruption of the tear film leads to increased corneal higher-order aberrations. ${ }^{34,35}$ These theories may explain why we found that there are unique environmental effects on corneal aberrations. Another potential explanation for the different heritability estimates between corneal and ocular aberrations is the effect of the crystalline lens. The lens thickness is more significant in ocular higher-order aberrations. Previously, the potential genetic influence on the crystalline lens has not been well established. However, recently, large studies have been conducted to estimate heritability for crystalline lens to suggest the possibilities of genetic effect on cataract formation. Lyhne et al. ${ }^{36}$ have previously reported that lens thickness is $90 \%$ to $93 \%$ heritable in Danish twins. Recently, Shen et al. ${ }^{37}$ have evaluated the heritability of lens thickness in 768 twin pairs in the Guangzhou Twin Eye Study. They report that ICCs from MZ and DZ twin pairs are 0.90 and 0.39 , respectively. The best-fitting model was AE with $89.5 \%$ of additive genetic effects and $10.5 \%$ of unique environmental effects. The study confirmed that lens thickness in young subjects is predominantly genetically determined, and that genetic factors may play a significant role in lens growth. Further evaluation is necessary to investigate the difference in heritability between corneal and ocular higher-order aberrations.

Our results suggest that spherical aberration has the highest heritability among ocular higher-order aberrations. Interestingly, spherical aberration also had relatively higher heritability among the lower values in corneal higher-order aberrations. 
TABLE 3. Heritability Estimates of Corneal and Ocular Higher-Order Aberrations*

\begin{tabular}{|c|c|c|c|c|c|}
\hline \multirow[b]{2}{*}{ Trait } & \multicolumn{3}{|c|}{ Variance Component } & \multirow[b]{2}{*}{ Best-Fitting Model } & \multirow[b]{2}{*}{ Variance of Covariates } \\
\hline & $\mathbf{A}$ & $\mathbf{C}$ & $\mathbf{E}$ & & \\
\hline \multicolumn{6}{|l|}{ Biometrics } \\
\hline Spherical equivalent, D & $0.74 \dagger$ & & 0.26 & $\mathrm{AE}$ & 0.253 \\
\hline Axial length, mm & $0.85 \dagger$ & & 0.15 & $\mathrm{AE}$ & 0.248 \\
\hline Anterior chamber depth, $\mathrm{mm}$ & $0.90 \dagger$ & & 0.10 & $\mathrm{AE}$ & 0.427 \\
\hline Corneal astigmatism, D & $0.50 \dagger$ & & 0.50 & $\mathrm{AE}$ & 0.042 \\
\hline \multicolumn{6}{|l|}{ Corneal aberration, $\mu \mathrm{m}$} \\
\hline \multicolumn{6}{|l|}{ Zernike coefficients } \\
\hline Vertical coma & $0.18 \dagger$ & & 0.82 & $\mathrm{AE}$ & 0.010 \\
\hline Horizontal coma & $0.31 \dagger$ & & 0.69 & $\mathrm{AE}$ & 0.018 \\
\hline Oblique trefoil & 0.05 & 0.03 & 0.91 & ACE & 0 \\
\hline Horizontal trefoil & $0.15 \dagger$ & & 0.85 & $\mathrm{AE}$ & 0.051 \\
\hline Spherical aberration & $0.18 \dagger$ & & 0.82 & $\mathrm{AE}$ & 0.009 \\
\hline Coma-like aberrations & 0.12 & 0.03 & 0.85 & ACE & 0.019 \\
\hline Spherical-like aberrations & $0.12 \dagger$ & & 0.88 & $\mathrm{AE}$ & 0.010 \\
\hline Third-order aberrations & 0.05 & 0.02 & 0.92 & ACE & 0.002 \\
\hline Fourth-order aberrations & $0.16 \dagger$ & & 0.84 & $\mathrm{AE}$ & 0.025 \\
\hline Fifth-order aberrations & 0.07 & 0.02 & 0.90 & ACE & 0 \\
\hline Sixth-order aberrations & $0.15 \dagger$ & & 0.85 & $\mathrm{AE}$ & 0.020 \\
\hline HoA & 0.02 & 0.07 & 0.91 & ACE & 0.012 \\
\hline \multicolumn{6}{|l|}{ Root-mean-square } \\
\hline Coma & 0.08 & & 0.92 & $\mathrm{AE}$ & 0.037 \\
\hline Trefoil & 0.06 & 0.01 & 0.93 & ACE & 0.043 \\
\hline Spherical aberration & $0.22 \dagger$ & & 0.78 & $\mathrm{AE}$ & 0 \\
\hline Coma-like aberrations & 0.08 & & 0.92 & $\mathrm{AE}$ & 0.045 \\
\hline Spherical-like aberrations & $0.17 \dagger$ & & 0.83 & $\mathrm{AE}$ & 0.003 \\
\hline Third-order aberrations & 0.04 & & 0.96 & $\mathrm{AE}$ & 0.051 \\
\hline Fourth-order aberrations & $0.19 \dagger$ & & 0.81 & $\mathrm{AE}$ & 0.031 \\
\hline Fifth-order aberrations & 0.04 & & 0.96 & $\mathrm{AE}$ & 0.041 \\
\hline Sixth-order aberrations & 0.08 & & 0.92 & $\mathrm{AE}$ & 0.058 \\
\hline HoA & 0.04 & & 0.96 & $\mathrm{AE}$ & 0.065 \\
\hline \multicolumn{6}{|l|}{ Ocular aberration, $\mu \mathrm{m}$} \\
\hline \multicolumn{6}{|l|}{ Zernike coefficients } \\
\hline Vertical coma & $0.39 \dagger$ & & 0.61 & $\mathrm{AE}$ & 0.026 \\
\hline Horizontal coma & $0.37 t$ & & 0.63 & $\mathrm{AE}$ & 0 \\
\hline Oblique trefoil & $0.31 \dagger$ & & 0.69 & $\mathrm{AE}$ & 0.014 \\
\hline Horizontal trefoil & $0.35 t$ & & 0.65 & $\mathrm{AE}$ & 0.030 \\
\hline Spherical aberration & $0.71 \dagger$ & & 0.29 & $\mathrm{AE}$ & 0.138 \\
\hline \multicolumn{6}{|l|}{ Root-mean-square } \\
\hline Coma & 0.09 & $0.09 \ddagger$ & 0.81 & $\mathrm{ACE}$ & 0.015 \\
\hline Trefoil & 0.07 & & 0.93 & $\mathrm{AE}$ & 0.081 \\
\hline Spherical aberration & $0.37 \dagger$ & & 0.63 & $\mathrm{AE}$ & 0.071 \\
\hline HoA & $0.34 \dagger$ & & 0.66 & $\mathrm{AE}$ & 0.170 \\
\hline Total aberration & $0.67 \dagger$ & & 0.33 & $\mathrm{AE}$ & 0.115 \\
\hline
\end{tabular}

A, additive genetic effects; C, common environmental effects; E, unique environmental effects.

* Adjusted for age, sex, age ${ }^{2}$, age-by-sex interaction, and age $^{2}$-by-sex interaction.

$\dagger P<0.001$.

$\neq P<0.05$.

There were sex differences of spherical aberration in both corneal and ocular higher-order aberrations. The results suggested that there are genetic influences on both corneal and ocular spherical aberrations.

The present study had several strengths. First, we comprehensively investigated the genetic influence on corneal and ocular higher-order aberrations and compared them. This investigation enabled us to understand the genetic influences and biological mechanisms of higher-order aberrations. Second, the extended family study including twins and their firstdegree relatives allowed us to calculate the narrow-sense heritability estimates. As compared to studies with the classic twin model including only $\mathrm{MZ}$ and $\mathrm{DZ}$ twins, ${ }^{8,9}$ we were able to rule out the potential risk of inflating the heritability by including extended family members. Another strength of our study was its statistical power derived from the large number of $\mathrm{MZ}$ and $\mathrm{DZ}$ twin pairs and family members included.

There were some limitations to the study. This study related to Korean individuals and might differ in other populations; in particular, most of the younger subjects are myopic, and potential cohort effects exist with these family-based data. 
The Healthy Twin Study has established that corneal higherorder aberrations have unique environmental influences, while ocular higher-order aberrations are moderately heritable in the Korean population. Compared to lower-order aberrations such as myopia and corneal astigmatism, which showed heritability between 0.70 and $0.78,{ }^{17}$ the heritability of higher-order aberrations are smaller, suggesting less genetic influences on higher-order aberrations. Both corneal and ocular spherical aberrations were genetically influenced with sex differences. Although ocular spherical aberrations also increased with age, it was likely because of the effect of aging on the crystalline lens. There had been no previous studies with large sample sizes that investigated the genetic and environmental contributions to both ocular and corneal higher-order aberrations. It is of great interest currently to correct higher-order aberrations to improve vision quality. Therefore, characterizing higherorder aberrations of human eyes helps to promote better understanding to improve visual performance in the future.

\section{Acknowledgments}

Supported by grants from the Korea Health Technology R\&D Project through the Korea Health Industry Development Institute (KHIDI, funding No. HI14C0064) and the National Research Foundation of Korea funded by the Korean Government (Nos. 2011-220-E00006 and 2010-0029113). The authors alone are responsible for the content and writing of the paper.

Disclosure: D.H. Lim, None; W. Kim, None; G. Han, None; G.H. Bae, None; M.H. Kim, None; M.J. Kim, None; Y.-M. Song, None; E.-S. Chung, None; T.-Y. Chung, None; J. Sung, None

\section{References}

1. Williams D, Yoon GY, Porter J, Guirao A, Hofer H, Cox I. Visual benefit of correcting higher order aberrations of the eye. $J$ Refract Surg. 2000;16:S554-S559.

2. Wang L, Dai E, Koch DD, Nathoo A. Optical aberrations of the human anterior cornea. J Cataract Refract Surg. 2003;29: 1514-1521.

3. Oshika T, Klyce SD, Applegate RA, Howland HC. Changes in corneal wavefront aberrations with aging. Invest Ophthalmol Vis Sci. 1999;40:1351-1355.

4. Guirao A, Redondo M, Artal P. Optical aberrations of the human cornea as a function of age. J Opt Soc Am A Opt Image Sci Vis. 2000;17:1697-1702.

5. Lombardo M, Lombardo G. Wave aberration of human eyes and new descriptors of image optical quality and visual performance. J Cataract Refract Surg. 2010;36:313-331.

6. Salmon TO, van de Pol C. Normal-eye Zernike coefficients and root-mean-square wavefront errors. J Cataract Refract Surg. 2006;32:2064-2074.

7. Fujikado T, Kuroda T, Ninomiya S, et al. Age-related changes in ocular and corneal aberrations. Am J Ophthalmol. 2004;138: 143-146.

8. Yeh LK, Chiu CJ, Fong CF, et al. The genetic effect on refractive error and anterior corneal aberration: twin eye study. $J$ Refract Surg. 2007;23:257-265.

9. Dirani M, Chamberlain M, Couper TA, Guymer RH, Baird PN. Role of genetic factors in lower- and higher-order aberrationsthe genes in myopia twin study. Ophthalmic Res. 2009; 41 : 142-147.

10. Morgan I, Rose K. How genetic is school myopia? Prog Retin Eye Res. 2005;24:1-38.

11. Dirani M, Chamberlain M, Garoufalis P, Chen C, Guymer RH, Baird PN. Refractive errors in twin studies. Twin Res Hum Genet. 2006;9:566-572.
12. Sanfilippo PG, Hewitt AW, Hammond CJ, Mackey DA. The heritability of ocular traits. Surv Ophthalmol. 2010;55:561583.

13. Dirani M, Shekar SN, Baird PN. Adult-onset myopia: the Genes in Myopia (GEM) Twin Study. Invest Ophthalmol Vis Sci. 2008; 49:3324-3327.

14. He M, Hur YM, Zhang J, Ding X, Huang W, Wang D. Shared genetic determinant of axial length, anterior chamber depth, and angle opening distance: the Guangzhou Twin Eye Study. Invest Ophthalmol Vis Sci. 2008;49:4790-4794.

15. Tsai MY, Lin LL, Lee V, Chen CJ, Shih YF. Estimation of heritability in myopic twin studies. Jpn J Ophthalmol. 2009; 53:615-622.

16. Morgan IG, Ohno-Matsui K, Saw SM. Myopia. Lancet. 2012; 379:1739-1748.

17. Kim MH, Zhao D, Kim W, et al. Heritability of myopia and ocular biometrics in Koreans: the Healthy Twin Study. Invest Ophthalmol Vis Sci. 2013;54:3644-3649.

18. Sung J, Cho SI, Lee K, et al. Healthy Twin: a twin-family study of Korea-protocols and current status. Twin Res Hum Genet. 2006;9:844-848.

19. Sung J, Cho SI, Song YM, et al. Do we need more twin studies? The Healthy Twin Study, Korea. Int J Epidemiol. 2006;35:488490.

20. Song YM, Lee D, Lee MK, et al. Validity of the zygosity questionnaire and characteristics of zygosity-misdiagnosed twin pairs in the Healthy Twin Study of Korea. Twin Res Hum Genet. 2010;13:223-230.

21. Thibos LN, Applegate RA, Howland HC, et al. VSIA-sponsored effort to develop methods and standards for the comparison of the wavefront aberration structure of the eye between devices and laboratories. In: Vision Science and Its Applications. Washington, DC: Optical Society of America; 1999: 236-239.

22. Thibos LN, Applegate RA, Schwiegerling JT, Webb R. Standards for reporting the optical aberrations of eyes. $J$ Refract Surg. 2002; 18:S652-S660.

23. Beiko GH, Haigis W, Steinmueller A. Distribution of corneal spherical aberration in a comprehensive ophthalmology practice and whether keratometry can predict aberration values. J Cataract Refract Surg. 2007;33:848-858.

24. Keller MC, Medland SE, Duncan LE, et al. Modeling extended twin family data I: description of the Cascade model. Twin Res Hum Genet. 2009;12:8-18.

25. Blangero J, Williams JT, Almasy L. Variance component methods for detecting complex trait loci. Adv Genet. 2001; 42:151-181.

26. Prakash G, Sharma N, Choudhary V, Titiyal JS. Higher-order aberrations in young refractive surgery candidates in India: establishment of normal values and comparison with white and Chinese Asian populations. J Cataract Refract Surg. 2008; 34:1306-1311.

27. Wei RH, Lim L, Chan WK, Tan DT. Higher order ocular aberrations in eyes with myopia in a Chinese population. $J$ Refract Surg. 2006;22:695-702.

28. Cantú R, Rosales MA, Tepichin E, Curioca A, Montes V, Bonilla $\mathrm{J}$. Whole eye wavefront aberrations in Mexican male subjects. $J$ Refract Surg. 2004;20:685-688.

29. Lim KL, Fam HB. Ethnic differences in high-order aberrations: spherical aberration in the South East Asian Chinese eye. J Cat Refract Surg. 2009;35:2144-2148.

30. Cerviño A, Hosking SL, Ferrer-Blaso T, Montes-Mico R, Gonzales-Meijome JM. A pilot study on the differences in wavefront aberrations between two ethnic groups of young generally myopic subjects. Ophthalmic Physiol Opt. 2008;28: 532-537. 
31. Cairns G, McGhee CN, Collins MJ, Owens H, Gamble GD. Accuracy of Orbscan II slit-scanning elevation topography. $J$ Cataract Refract Surg. 2002;28:2181-2187.

32. Cairns G, McGhee CN. Orbscan computerized topography: attributes, applications, and limitations. J Cataract Refract Surg. 2005;31:205-220.

33. Carvalho LA. Absolute accuracy of Placido-based videokeratographs to measure the optical aberrations of the cornea. Optom Vis Sci. 2004;81:616-628.

34. Koh S, Maeda N, Kuroda T, et al. Effect of tear film break-up on higher-order aberrations measured with wavefront sensor. $\mathrm{Am}$ J Ophthalmol. 2002;134:115-117.
35. Lin YY, Carrel H, Wang IJ, Lin PJ, Hu FR. Effect of tear film break-up on higher order aberrations of the anterior cornea in normal, dry, and post-LASIK eyes. J Refract Surg. 2005;21: S525-S529.

36. Lyhne N, Sjolie AK, Kyvik KO, Green A. The importance of genes and environment for ocular refraction and its determiners: a population based study among $20-45$ year old twins. $\mathrm{Br}$ J Ophthalmol. 2001;85:1470-1476.

37. Shen P, Ding X, Zheng Y, Congdon NG, He M. Contribution of genetic and environmental effects on lens thickness: the Guangzhou Twin Eye study. Invest Ophthalmol Vis Sci. 2012; 53:1758-1763. 